\title{
Biological Risks and their Communication for Public
}

\section{N.V. Shadrin*}

Institute of Biology of the Southern Seas, Sevastopol, Ukraine

Increasing of biological \& ecological risks is a global threat to human life, livelihoods and natural life-supporting systems. No one can be safe from them. One of main reasons of such situation is an inadequate management of our activities, which effectively multiplies these risks making few for a prevention and mitigation of them. Discussing why we have such inadequate management we need to remember that we simultaneously live and operate in two worlds - the world of physical reality and the world of signs/symbols. Our interaction with the real physical world is mediated by our activity in the sign world. The world of signs includes all our traditions, concepts, goals, motivations, and models of the world including our perception and weighted values of different biological and ecological dangers. As usual the dangers as well as our management activities are in the real physical world, but all our decisions and plans are made in the world of signs. Reasonableness of our operation in the world around us is determined primarily by how adequately it is reflected in our sign-world, i.e. as far as our concepts and paradigms are similar to the physical world around us in its variability. In the public perception there are a lot of underestimated risks but other ones are overweighed. It's a result of media dependency of the public mind and poor fundamental knowledge of journalists on bio-ecosafety issues. Many such issues are also not enough clear for understanding by scientists. To go towards an effective prevention and mitigation of bio/ecorisks we need to solve two main problems - to get an adequate scientific knowledge and to communicate its understanding to the public. The adequate understandings of risks by the public can lead to political and economical solutions to solve the problems. I hope new Journal of Biosafety \& Health Education - Open Access will make a real contribution to solve both problems being an interdisciplinary and intersectoral platform for new data, ideas, and discussions. And the complementarity principle - there is no single best description/model of any system - will be among its governing rules. Currently a most part of biosafety and ecosafety problems are studied and discussed separately. But they are interconnected. It's impossible task to list all such couplings, but some of them I'll touch.

\section{Potentially Toxic Cyanobacteria}

Cyanobacteria are oxygenic phototrophs, the first on Earth; they live in a variety of extreme conditions. All this resulted to that they are producers of various secondary metabolites, including biotoxins and cytotoxins that affect animals and humans. Toxins of cyanobacteria are grouped in two main categories: biotoxins and cytotoxins based on the types of bioassays used to screen for their activity. As a result of increasing anthropogenic pollution and global warming the problem of mass development of cyanobacteria in water bodies has become global. Outbreaks of human poisoning attributed to toxic cyanobacteria have been reported in many countries. Blooming of cyanobacteria in water bodies is a result of aquatic ecosystem changes - transit of ecosystems from one stable state to other alternative one. Can we predict aquatic ecosystem changes leading to cyanobacteria blooming? Potentially toxigenic species not always produce toxins, but produce only in some cases. What environmental factors can lead to switching of cyanobacteria from one state to other one - from non toxic to toxic? Global warming leads to salinization of many water bodies. It is known that salinity can influence on the expression of some genes. We need to know how salinity influences on expression of genes responsible for synthesis of toxins. Environmental changes can influence on toxigenity and virulence of many microorganisms. We need to know how, and this point is coupling of ecology, epidemiology, and biosafety.

\section{Anthropogenic Impact on Ecosystems and New Host- Parasite Systems}

People destroy different landscapes and promote introducing of alien species. Both reasons lead to rearrangement of species composition of natural communities with creation of new hostparasite systems and new parasitic cycles sometimes with an inclusion of man. Global climate changes also can lead to such results. Do we know enough about such transformations and their consequences for the public biosafety?

\section{Multiplicity of Alternative Stable States of Ecosystems, Population, and Individuals}

The biological / ecological systems at all levels - from molecular to ecosystem, as a rule, have not one but several alternative stable states. Realization of this is the essence of a new paradigm in biology and ecology, instead of one that considers the biological / ecological systems as having one point of global stability, and all changes of the system are only the fluctuations around this point. Now new paradigm is well accepted in genetics - epigenetics, but makes only first steps in ecology. We don't describe this concept here, but remember: destabilization of system leads it to tipping point, after the passage of which the system can not return to the previous stable state and will inevitably be transformed into a new state. Health ecosystem, health individual, health population can jump in unhealthy states. Transformations on all levels are interconnected - principle of panarchy. We need to know about these interconnections to create an effective system of public biosafety. New perspectives for rational management of the public biosafety require paradigm shift.

\section{Risk Communication}

So often we can see such logics "knowledge create motives for behavior, and we need only to educate people". Is it true in all cases? No, a lot of people know that to smoke is danger for health and continue to smoke. How knowledge transforms in behavioral motives? And other issue - public and scientific languages are quite different. What effective interface for communication between science and public do we need to develop?

*Corresponding author: N.V. Shadrin, Institute of Biology of the Southern Seas, Sevastopol, Ukraine, E-mail: snickolai@yandex.ru

Received October 19, 2012; Accepted October 19, 2012; Published October 21, 2012

Citation: Shadrin NV (2012) Biological Risks and their Communication for Public J Biosafety Health Educ 1:e101. doi:10.4172/2332-0893.1000e101

Copyright: (c) 2012 Shadrin NV. This is an open-access article distributed unde the terms of the Creative Commons Attribution License, which permits unrestricted use, distribution, and reproduction in any medium, provided the original author and source are credited. 